\title{
Influência do Treinamento Presencial e à Distância (EAD) sobre o Desempenho Individual dos Gestores
}

\section{Influence of Presence and Distance Training on the Managers' Individual Performance}

\author{
Marcos Paulo Albarello Friedrich \\ Doutorando em Contabilidade e Administração-Universidade Regional de Blumenau - FURB \\ Rua Antonio da Veiga, 140 - Itoupava Seca, CEP: 89030-903 - Blumenau-SC \\ E-mail: marcos.ensino@gmail.com
}

\author{
Alexandre Corrêa dos Santos \\ Doutorando em Contabilidade e Administração-Universidade Regional de Blumenau - FURB \\ Rua Ametista, 289 - Royal Boulevard Residence, CEP: 86.200-000 - Ibiporã-PR \\ E-mail: acs@ atuaris.com.br \\ Nelson Hein \\ Doutor em Engenharia de Produção - UFSC \\ Professor do PPGCC da Universidade Regional de Blumenau - FURB \\ Rua Antonio da Veiga, 140 - Itoupava Seca, CEP: 89030-903 - Blumenau-SC \\ E-mail: hein@furb.br
}

\begin{abstract}
Resumo: A aprendizagem organizacional (AO) é uma importante fonte de vantagem competitiva para as organizações, pois representa uma ponte entre as estratégias de negócio e o desenvolvimento dos colaboradores. Este estudo investiga a relação entre os treinamentos presenciais $(\mathrm{TP})$ e à distância $(\mathrm{EAD})$ de uma universidade corporativa e o desempenho sob a ótica da perspectiva cognitiva de aprendizagem na organização. Neste contexto, foi realizado um estudo de caso único com dados primários coletados junto ao departamento de recursos humanos da instituição pesquisada no período de 12 a 13 de junho de 2018 referentes à carga de treinamento em horas-aula para as duas modalidades. Utilizou-se os métodos de análise multicritério CRitic e TOPSIS para determinar os pesos de cada indicador de resultado individual e ranquear o desempenho dos profissionais analisados e regressão linear para identificar os efeitos de cada modalidade de desempenho. Os achados apontam que nos dois grupos de gestores os treinamentos presenciais apresentaram resultados negativos sobre o desempenho individual, enquanto os treinamentos à distância mostraram resultados positivos para os gestores pessoa jurídica e negativos para pessoa física.
\end{abstract}

Palavras-Chave: Universidade Corporativa; Treinamento Presencial; Treinamento à Distância.

Abstract: Organizational learning (OL) is an important source of competitive advantage for organizations, as it represents a bridge between business strategies and employee development. This study investigates the relationship between presence and distance training of a corporate university and the performance from the perspective of the cognitive learning in the organization. In this context, a single case study was carried out with primary data collected from the human resources department of the institution researched from June 12 to June 13, 2018, regarding the training load in class hours for the two modalities. The CRitic and TOPSIS multicriteria analysis methods were used to determine the weights of each individual outcome indicator and to rank the performance of the professionals analyzed and linear regression to identify the effects of each performance modality. The findings indicate that, in both groups of managers, presence training presented negative results on individual performance, while distance training showed positive results for corporate and negative for individuals managers. 
Keywords: Corporate University; Presence Training; Distance Training.

\section{INTRODUÇÃO}

A segunda metade do século XX foi marcada por uma série de mudanças no que diz respeito a educação corporativa no Brasil. Segundo Eboli (2011) ao mesmo tempo em que se desenvolviam atividades de treinamento e capacitação, necessidades de resposta à problemas de produtividade passaram a fazer parte de seus objetivos.

Entre 1970 e 1980, o cenário empresarial brasileiro sofre fortes mudanças, o conhecimento passa a ser considerado como capital e fonte de recursos, as organizações passam a ser mais flexíveis devido à necessidade de respostas rápidas ao mercado (MEISTER, 1999). O foco dos controles sai dos processos e passa para os resultados (MALVEZZI, 1994) e as discussões acerca do termo competência começam a ganhar corpo dentro do contexto organizacional (FLEURY e RUAS, 2008). Nesse período de profundas mudanças e necessidade de adaptação das organizações ao seu contexto, surgem as primeiras Universidades Corporativas (UCs) em substituição aos departamentos de T\&D. Seu objetivo passa a ser formar e desenvolver talentos de forma contínua em toda a organização através de competências empresariais e humanas que contribuam para a aprendizagem organizacional (AO) e para os resultados da empresa também no longo prazo.

Nesse contexto, a aprendizagem organizacional pode ser definida como um processo de aquisição, compartilhamento, interpretação e uso do conhecimento (IMAMOGLU, INCE, KESKIN, KARAKOSE e GOZUKARA, 2015) com o objetivo de melhorar as ações e resultados ao longo do tempo (ARGOTE e MIRON-SPEKTOR, 2011; LEE, RITTINER e SZULANSKI, 2015). A literatura de estudos econômicos busca investigar a importância da (AO) como pilar para alcançar diferencial competitivo e continuidade em mercados globalizados (SCHARDOSIM, GOMES JR., TORQUATO e LAPOLLI, 2017; BOERNER, MACHER e TEECE, 2001), bem como sua relação com inovação ambiental (JACOMOSSI e DEMAJOROVIC, 2017; PAVITT, 1987).

Não esgotadas todas as abordagens de estudo da (AO) por não ser objeto deste trabalho, neste estudo investiga-se a relação entre universidade corporativa e desempenho sob a ótica da perspectiva cognitiva de aprendizagem na organização, em que se enfocam experiências práticas bem-sucedidas e centra-se em condutas que as organizações devem seguir para aprender (DAVEL e OLIVEIRA, 2018; ARAUJO e EASTERBY-SMITH, 2001).

Recentemente, mais especificamente nos últimos 10 anos, a busca pela aprendizagem organizacional nas UCs vem se modificando, principalmente com o avanço da educação a distância (EAD). Segundo Brauer e Albertin (2010) essa forma de educação avança juntamente com a tecnologia e com as rotinas de trabalho cada vez mais aceleradas. Esses autores afirmam que pesados investimentos vêm sendo realizado na educação a distância corporativa para implementação e desenvolvimento de cursos nessa modalidade. Entretanto, a EAD Corporativa, além de todos os desafios dos treinamentos tradicionais, enfrenta ainda as dificuldades relacionadas ao uso e aceitação da tecnologia, além de limitações quanto a interação dos usuários por realizarem os cursos em horários flexíveis (MUNGANIA, 2003).

Porém, Zerbini, Nascimento, Meneses e Abbad (2006) afirmam que, apesar de todo o investimento realizado pelas grandes corporações em busca da aprendizagem organizacional, sua relação com o desempenho organizacional ainda não está consolidada. Para esses autores, isso se deve ao fato de que, em 1990, os treinamentos eram tratados apenas como estratégias remediadoras e recentemente estão sendo alterados para uma roupagem mais proativa, de forma a alcançar uma vantagem competitiva sustentável (DAVENPORT e PRUSAK, 1998). Desse modo, tanto os treinamentos presenciais quanto aqueles à distância, ainda carecem de uma relação mais consistente com o desempenho organizacional para comprovar seu alinhamento com as estratégias propostas pela empresa. 
Neste contexto, Antonello e Godoy (2010) explicam que, independente da modalidade de ensino, estabelecer um link entre aprendizagem organizacional e desempenho individual é um grande desafio, pois, de forma geral, assume-se uma relação positiva, contudo, de modo trivial e talvez até superficial, sem sustentação na literatura.

Diante dessa lacuna, esse estudo se propõe a responder a seguinte questão de pesquisa: Qual o impacto dos treinamentos EAD e presencial de uma universidade corporativa no desempenho individual dos gestores da empresa?

$\mathrm{Na}$ busca de solucionar este problema, realizou-se um estudo de caso em uma instituição financeira que possui um departamento de treinamento há mais de 50 anos, recentemente transformado em Universidade Corporativa e que, nos últimos cinco anos, passou a adotar também cursos EAD em seu portfólio.

Investigar esta questão contribui cientificamente para mitigar o senso comum de que empresas que possuem as chamadas "universidades corporativas" obterão melhor desempenho dos gestores apenas pela existência do programa, irrelevante às contrapartidas oferecidas ao gestor. De outra via, fornece um meio de investigar se a concepção de que tais programas estão mais ligados à aprendizagem organizacional, ou seja, um ativo que pode gerar informações a longo prazo para a organização, independentemente da permanência dos gestores.

Esse estudo contribui para a literatura referente à educação à distância corporativa não somente avaliando fatores de resistência (BRAUER e ALBERTIN, 2010), fatores chave de sucesso (JOIA e COSTA, 2007) ou limitações e restrições da implementação (ZERBINI et al., 2006), como também os efetivos resultados dessa modalidade de treinamento e suas diferenças para os resultados dos treinamentos presenciais.

Do ponto de vista prático, o estudo possibilita uma melhor compreensão da eficácia dos programas de treinamento interno e sua capacidade de gerar conhecimento estratégico para a organização, ajuda as organizações a discernirem a criação de manutenção de tais programas ou investir recursos em outra forma de aprendizagem organizacional.

A sequência deste trabalho está dividida em cinco seções, sendo: introdução; referencial teórico, que é subdividido em: universidades corporativas e a educação a distância, competências gerenciais versus desempenho do gestor, procedimentos metodológicos; análise e discussão dos resultados e considerações finais.

\section{REVISÃO DA LITERATURA}

\subsection{Universidades Corporativas e a Educação a Distância}

Os desafios advindos da globalização econômica mundial exigem das organizações a condução de processos de gestão mais dinâmicos e flexíveis. Para que isto seja viabilizado, a informação prioritária nas organizações é transmitida aos colaboradores por meio de programas educação corporativa. Segundo Esteves e Meiriño (2015) a educação corporativa é a junção coordenada de gestão de pessoas e do conhecimento com foco na otimização das estratégias organizacionais.

Nesta direção, Leal (2014) descreve uma linha do tempo para os programas de treinamento em nível corporativo buscando compreender as transformações ocorridas diante do contexto empresarial da época. Segundo esse autor, até 1950, o objetivo dos treinamentos era apenas operacional do tipo "aprender fazendo", pois, as tarefas dos trabalhadores eram simples e repetitivas. Somente em 1950, surge a definição de treinamento e desenvolvimento (T\&D) para a qual o treinamento passa a referir-se a aprendizagem de curto prazo enquanto o desenvolvimento ao processo de longo prazo, com foco na expansão do potencial dos trabalhadores.

A partir da década de 70, surgem as primeiras discussões acerca do conceito de competência e uma série de mudanças no cenário começam a transformar o trabalho, neste contexto, as organizações são mais flexíveis e exigem respostas rápidas ao ambiente de 
concorrência, o conhecimento torna-se a base da riqueza e do capital das organizações, ocorre rápida obsolescência do conhecimento e surge a necessidade de formar pessoas com visão global dos negócios (MEISTER, 1999).

Somente em 1980, aparecem as primeiras Universidades Corporativas (UCs) em substituição aos departamentos de T\&D. Seu objetivo passa a ser formar e desenvolver talentos de forma contínua em toda a organização através de competências empresariais e humanas que contribuam para a aprendizagem organizacional (AO) e para os resultados da empresa também no longo prazo.

Tais organizações apresentam uma natureza híbrida e pode ser referida tanto como uma unidade de negócios ou como uma instituição de ensino superior, cuja função busca conectar os processos de aprendizagem com os objetivos estratégicos, permitindo que as empresas promovam conhecimento e educação geral, enquanto que, ao mesmo tempo, incorporam conteúdos no contexto organizacional (CAPPIELLO e PEDRINI, 2017).

Nesta linha, Lytovchenko (2016) explica que a (UC) é uma forma avançada de treinamento organizacional que surgiu em resposta aos desafios da sociedade da informação e da economia global, do aumento da concorrência nos mercados e do fracasso das instituições tradicionais de educação profissional para atender às necessidades da economia moderna em manter funcionários altamente qualificados.

Todavia, o termo é usado como uma metáfora, pois está relacionado aos processos de aprendizagem que ocorrem dentro das organizações, dadas as iniciativas de aprendizagem e para aumentar os programas educacionais das organizações (CASTRO e EBOLI, 2013; MAGLIONE e PASSIANTE, 2009). Portanto, a terminologia UC é utilizado para dar ao projeto uma conotação de grandeza da iniciativa, cuja finalidade é o aprimoramento contínuo para o sucesso no trabalho na carreira organizacional (RUGGIERO, 2006).

Dada a dificuldade de conceituação, a forma mais comum de caracterizar uma (UC), é representá-la por meio de sua forma de atuação. A partir disso, (Allen, 2002, p.9) a caracteriza "como uma entidade educacional que é uma ferramenta estratégica designada a auxiliar sua matriz no alcance de sua missão pela condução de atividades que cultivam aprendizagem individual e organizacional, conhecimento e sabedoria".

Além disso, segundo Castro e Eboli (2013) o que caracteriza a educação corporativa é sua vinculação estratégica com a vida da empresa, representada pela capacitação contínua de pessoas dentro das organizações para atender as necessidades do mercado, adquirindo diferenciais competitivos (RUGGIERO, 2015).

Quanto à função organizacional das UCs, Abel e Li (2012) enfatizam que as mesmas facilitam as metas organizacionais e objetivos através da aquisição, gestão e retenção de talentos, bem como desempenham uma função importante para facilitar a aprendizagem (WANG et al., 2010).

Na opinião de Meister (1999) as UCs surgiram de algumas forças como, a flexibilidade das organizações, as novas formas de conhecimento, a rápida obsolescência do conhecimento, questões ligadas a empregabilidade e uma nova forma e educação para estratégia global.

Portanto, diante de sua importância, Alagaraja e Li (2015) enfatizam que as UCs não podem ser descartadas como uma moda passageira, pois ilustram a evolução e a transformação dos departamentos tradicionais de treinamento e desenvolvimento em novas formas institucionais ao longo do tempo.

Mais recentemente, as UCs migraram para um formato virtual facilitado pelo desenvolvimento de tecnologias digitais, com maior ênfase no alinhamento do treinamento corporativo e pessoas com os objetivos estratégicos da organização, com o fim de formação de lideranças sobre as áreas funcionais (RYAN, PRINCE e TURNER, 2015). A Figura 1 mostra dados da pesquisa de Eboli (2016) que aponta as principais razões elencadas pelas Universidades Corporativas para uso do EAD. 


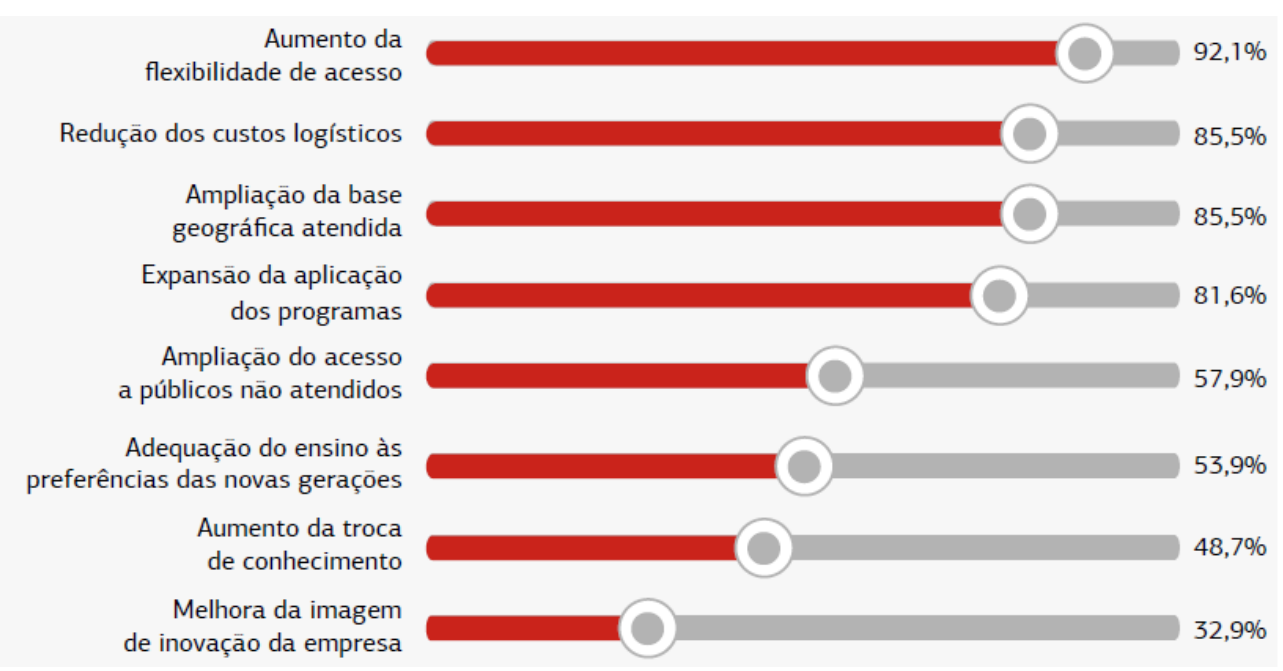

Figura 1: Principais motivos para utilizar a educação a distância

Fonte: adaptado de Eboli (2016).

Neste sentido, (DURAN 2017; MACHADO e MORAES, 2015) corroboram ao afirmar que o formato virtual insere os indivíduos em um ambiente de avanços tecnológicos, portanto, configura um espaço de democratização da educação, de interação e rompimento das barreiras espaço-temporais. Além disso, a imagem afetiva dos alunos está ligada de forma significante ao fator ambiente virtual de aprendizagem (AVA), aliado à qualidade do ensino (COSTA e PELISSARI, 2017). De outro lado, para as organizações o treinamento corporativo com foco EAD contribui para a otimização dos recursos, a redução de custos, considerando-se a possibilidade de treinar diversos colaboradores em localidades diversas, representando um elo entre o desenvolvimento de pessoas e a visão estratégica da organização (MOSCARDINI e KLEIN, 2015).

Contudo, no caso brasileiro, as pesquisas neste campo indicam que a prática de educação corporativa ainda está em evolução, considerando que muitas empresas não conseguem implementar de forma prática os princípios presentes nos estudos dos principais estudiosos desta área (VIDAL, 2017).

Enquanto práticas, as estratégias de ensino e aprendizagem mais utilizadas são aulas expositivas e debates, em que a modalidade presencial predomina e a utilização das tecnologias de informação e comunicação são limitadas ao uso de Ambientes Virtuais de Aprendizagem e ferramentas da Web 2.0 (MOSCARDINI e KLEIN, 2015)

Neste contexto, há ainda um longo caminho de investigações no campo das pesquisas para que se possa alcançar respostas mais conclusivas sobre o futuro das universidades corporativas e, de fato, como e por quais meios esses institutos interagem com as competências gerenciais e no desempenho econômico das organizações.

\subsection{Competências Gerenciais versus Desempenho Individual do Gestor}

Nas últimas décadas, as organizações apoiaram-se em sistemas de informações gerenciais com funções de mensuração de desempenho dos gestores (Hall, 2008). Neste contexto, as escolhas racionais de avaliação de desempenho gerencial nas organizações precisam levar em conta fatores controláveis e incontroláveis, em que os primeiros podem remeter a um estado de menor risco em relação aos últimos, afetar positivamente o nível de motivação, evitar comportamentos disfuncionais e míopes e gerar a criação de reservas (BEUREN, ROTH e ANZILAGO, 2017; MERCHANT e VAN DER STEDE, 2007).

Por sua vez, os fatores incontroláveis, segundo Simons (2005) podem desempenhar papel importante para o gestor, à medida que, ao estender a responsabilidade dos gestores para 
fatores sem controle absoluto, incentivam/estimulam a inovação e o comportamento empreendedor.

Neste contexto, os programas de aprendizagem organizacional criados sob uma perspectiva de negócios e desenvolvimento pessoal dos gestores, precisam ser estrategicamente delineados, de tal forma que, a organização alcance seus objetivos junto aos clientes e, internamente, mantenha a motivação gerencial e o seu desenvolvimento humano e social permanente.

Para alcançar este fim, Silva e Freire (2017) comentam que os modelos mais atuais de UCs mapeados na literatura correspondem ao um sistema em rede (UCR) que é conduzido por sete diretrizes (alcance, interconexão, reconhecimento dos stakeholders, tecnologia, foco, nível e gestão do conhecimento) que direcionam e estruturam as ações corporativas. Portanto, este modelo remete à participação mais integrada e efetiva da área de gestão de pessoas, o que alinha ações de desenvolvimento dos gestores ao mapa estratégico da organização.

Nesta linha, pode-se inferir que um dos caminhos que leva a melhor desempenho dos gestores passa pelo desenvolvimento de suas competências, as quais a organização pode ajudar a construir em sua universidade corporativa. Neste sentido, Freitas e Odelius (2018) confirmam que dentre as categorias de competências gerenciais presentes na literatura, destacam-se aquelas com orientação para resultados (clientes, processos, custos, mercado, produtos), gestão do conhecimento, conhecimentos e habilidades técnicas da área, dentre outros.

No entanto, o que se observa é que na literatura o termo competência agrega vários significados, tanto ligados às características dos indivíduos, ou relacionado a função ou cargo por ela exercido e agrega elementos subjetivos, como os conhecimentos, habilidades e atitudes (FLEURY e RUAS, 2008). Para fins deste estudo, busca-se um alinhamento entre as questões subjetivas e próprias dos indivíduos e a forma como a competência é utilizada tendo como fim os objetivos estratégicos das organizações (GONÇALVES et al., 2017).

Tal alinhamento pode ser melhorado por meio dos processos de aprendizagem, que desempenham papel relevante no desenvolvimento de capacidades e competências organizacionais e constituem uma questão recorrente no debate sobre estratégia e competividade (NASCIMENTO, 2017; EASTERBY-SMITH e PRIETO, 2008; HELFAT e PETERAF, 2009).

Pesquisas mais recentes em instituições financeiras, como a de Avelino (2016) mostram que a relação entre as competências gerenciais mapeadas pela organização e as demandas na execução das atividades são positivamente relacionadas nas unidades de alta performance.

Neste contexto, considerando-se o atual estágio de desenvolvimento das universidades corporativas no Brasil e a crescente necessidade por competências gerenciais avançadas orientadas para resultados, levantam-se as seguintes hipóteses de pesquisa:

H1 A universidade corporativa, por meio de seus treinamentos presenciais, influencia positivamente o desempenho dos colaboradores.

H2 A universidade corporativa, por meio de seus treinamentos à distância (EAD), influencia positivamente o desempenho dos colaboradores.

\section{PROCEDIMENTOS METODOLÓGICOS}

Para atingir o objetivo proposto e testar as hipóteses, realizou-se uma pesquisa descritiva de abordagem quantitativa, caracterizada como um estudo de caso único que, segundo Yin (2015) pode ser usado para contribuir para o conhecimento dos fenômenos individuais, grupais, organizacionais, sociais, políticos e relacionados. Esse método permite ao pesquisador focar em um caso e obter uma perspectiva útil para as demais organizações. 


\subsection{População e Amostra}

A empresa alvo do estudo possui 90 anos de existência e atuação regional, apresenta estrutura com 528 unidades, envolve 11.050 colaboradores e abrange 7 Estados brasileiros em cerca de 500 municípios. Essa organização foi escolhida por conveniência, pois apresenta características que se relacionam ao objetivo do estudo em uma universidade corporativa com sistema de mensuração de desempenho individual de seus gestores.

Em agosto de 2017 a instituição celebrou 51 anos de educação corporativa e, frente ao amadurecimento dessa trajetória, decidiu em 2014, abandonar o conceito de gerência de treinamento para assumir o conceito de Universidade Corporativa, com o objetivo de orientarse de forma mais intensa para a qualidade do processo de ensino-aprendizagem e fortalecer a qualificação de seus profissionais.

Com uma extensa presença geográfica, as dificuldades para manter os treinamentos presenciais corporativos são um constante desafio, por outro lado, o dinamismo dos mercados nos quais está inserida, exige constante atualização tecnológica e de processos para a execução dos negócios, o que demandou a implementação de outras modalidades de treinamento.

Em 2013, a então gerência de treinamento, hoje Universidade Corporativa, lançou uma plataforma de ensino à distância por meio do Moodle para promover e divulgar cursos, treinamentos e atualizações, de modo que todos os funcionários tivessem oportunidade de acessar o conteúdo dos cursos e não somente aqueles selecionados para os treinamentos presenciais. Inicialmente a plataforma dispunha de apenas 3 cursos, hoje conta com mais de cem nas mais diversas áreas (técnica, comportamental, dentre outras). Após cinco anos em funcionamento, mais de 1 milhão de horas em cursos já foram registradas na plataforma EAD.

Do total de colaboradores, optou-se por aqueles que possuem avaliação individual tanto de desempenho comportamental quanto comercial para que se pudesse medir o impacto do treinamento presencial e EAD em seus resultados. A amostra contou com 1177 gestores, em que 905 são gerentes pessoa física (67\% do sexo feminino) e 272 gerentes pessoa jurídica (57\% do sexo feminino) que representam $100 \%$ dos funcionários que atuam nessas funções na empresa. Desse total, foram excluídos da amostra final 82 gestores pessoa física e 8 gestores pessoa jurídica, por estarem em período de estágio na função e/ou não apresentarem dados para avaliação de desempenho individual na função, totalizando uma amostra final de 1087 (823 PF e 264 PJ). O critério de escolha ocorre porque esses colaboradores gerenciam carteiras de clientes de forma individual e seu desempenho depende exclusivamente de sua atuação.

\subsection{Coleta e Tratamento dos Dados}

Os dados foram coletados em visita à Universidade Corporativa da empresa nos dias 12 e 13 de junho de 2018. A UC é responsável por desenvolver um ranking de gestores (Índice de Desempenho - ID) a cada divulgação dos resultados semestrais, que serve como base para processos seletivos internos. Foram utilizados todos os dados formadores do índice, a saber: i) pontuação na meta comercial média de 4 semestres (formada pelo volume multiplicado pela margem de contribuição líquida de cada produto; ii) pontuação da meta estratégica média de 4 semestres (formada pela quantidade de venda em produtos específicos multiplicada pela pontuação de cada produto determinada pela ordem de importância de cada um na estratégia da empresa; iii) pontuação média de 4 semestres do indicador de gestão de despesas da carteira; iv) pontuação no semestre da adimplência de crédito da carteira; v) pontuação formada pela avaliação comportamental realizada pelo superior direto; vi) pontuação do compliance e auditoria física de documentos e contratos.

Além desses dados, coletou-se também dados referentes ao gênero, idade, tempo na empresa, horas de treinamento em EAD e presencial e, o último índice de desempenho (ID) divulgado pela empresa em março 2018 referente ao semestre julho a dezembro 2017. 


\subsection{Procedimentos de Análise dos Dados}

Inicialmente, utilizou-se o Technique for Order Preference by Similarity to na Ideal Solution (TOPSIS) para classificar cada gestor de acordo com seu desempenho. A técnica TOPSIS, de acordo com Tzeng e Huang (2011) é um modelo de análise multicritério que classifica os dados em relação à sua distância do melhor (mais próximo da solução ótima) e do pior (mais distante da solução ótima) desempenho dentro da amostra apresentada. O resultado do TOPSIS é um número entre 0 e 1 em que, quanto mais próximo de 1 melhor, ou seja, no caso do presente estudo, gestores com resultado mais próximo de 1 apresentam melhor desempenho entre os dados analisados.

Dentro do conjunto de seis critérios apresentados para classificação que são pontuações na meta comercial, na meta estratégica, na gestão de despesas, na adimplência de crédito da carteira, formada pela avaliação comportamental realizada pelo superior direto e do compliance e auditoria física de documentos e contratos, foi necessário identificar-se o peso de cada um deles para a análise multicritério. Os pesos foram obtidos por meio do método CRITIC, acrônimo de Criteria Importance Through Intercriteria Correlation, desenvolvido por Diakoulaki, Mavrotas e Papayannakis (1995), que utiliza os conceitos de desvio padrão das variáveis em uso e a correlação entre as mesmas.

O peso de cada um dos j critérios é calculado por meio da expressão de normalização $w_{j}=\frac{C_{j}}{\sum_{k=1}^{c} C_{k}} ; j=1,2,3$. O valor de cada $C_{j}$ é dado por: $C_{j}=\sigma_{j} \sum_{k=1}^{c}\left(1-r_{j k}\right)$, em que $\sigma_{j}$ é o desvio-padrão da variável j e $r_{j k}$ é o coeficiente de correlação da variável j com as duas demais variáveis em análise. Pelo método CRITIC os pesos dos critérios são afetados tanto pelas características dos critérios quanto pelo ponto de vista subjetivo do decisor. Com isso, tanto o desvio padrão do critério quanto sua correlação entre outros critérios são incluídos no processo de ponderação.

Mediante essas informações, os resultados da análise TOPSIS permitiram classificar os gestores de acordo com seu desempenho. Desse modo, foram gerados dois rankings (pessoa física e pessoa jurídica) em que o primeiro colocado foi o que apresentou desempenho mais próximo do considerado ideal positivo (solução ótima) e mais distante do considerado ideal negativo (solução inferior), (BULGURCU, 2012).

Por fim, para responder o problema de pesquisa e testar as hipóteses propostas, realizouse duas regressões lineares (pessoa física e jurídica) para cada ranking por meio do software Statistical Package for the Social Sciences (SPSS), versão 23®. Utilizou-se a regressão linear porque possibilita analisar a relação entre uma ou mais variáveis explicativas, que se apresentam na forma linear e uma variável dependente quantitativa (FÁVERO e FÁVERO, 2016). O objetivo desse procedimento foi de mensurar o quanto cada uma dessas variáveis independentes influencia no desempenho dos gestores. Desse modo, os modelos de regressão linear utilizados podem ser descritos na forma da equação (1), sendo a variável "sexo" utilizada apenas para controle.

$$
\begin{array}{r}
\text { TOPSIS }_{i}=\beta_{0 i}+\beta_{1} I_{i}+\beta_{2} \text { Sexo }_{i}+\beta_{3} \text { Idade }_{i}+\beta_{4} \text { TempoEmp }_{i}+\beta_{5} \text { HorasEAD }_{i}+ \\
\beta_{6} \text { HorasPresen }_{i}+\epsilon_{i}
\end{array}
$$

Em que:

TOPSIS: representa o ranking desenvolvido nessa pesquisa através do método CRitic e Topsis. $I D$ : representa o indicador de desempenho utilizado pela instituição.

Sexo: sexo dos gestores da amostra.

Idade: idade dos gestores da amostra.

TempoEmp: tempo de serviço de cada colaborador na empresa.

HorasEAD: Horas de treinamento em EAD. 
HorasPresen: Horas de treinamento presencial.

$i$ : amostra específica de gestores pessoa jurídica ou física.

$\epsilon$ : erro amostral.

Os dados coletados foram tabulados em planilhas eletrônicas com utilização do software Excel@, a fim de organizá-los para os cálculos propostos. Na etapa de análise dos dados, inicialmente é apresentada a estatística descritiva das variáveis e posteriormente são apresentados os resultados e sua discussão

\section{APRESENTAÇÃO E ANÁLISE E DOS RESULTADOS}

Esta sessão apresenta os resultados encontrados na presente pesquisa e uma breve discussão dos mesmos em comparação com a literatura pertinente. São analisadas as estatísticas descritivas, o ranking TOPSIS e a Regressão Linear com foco na solução do problema de pesquisa proposto.

\subsection{Gerentes Pessoa Jurídica ( $\mathrm{N}=\mathbf{2 6 4})$}

Os dados apresentados na Tabela 1 mostram os 10 melhores (mais próximos de 1) e os 10 piores (mais distantes de 1) entre os 264 gestores pessoa jurídica analisados através do método TOPSIS, assim como o total de horas dedicadas a treinamentos na modalidade EAD e na modalidade presencial.

Tabela 1 - Ranking dos gestores pessoa jurídica baseado na técnica TOPSIS

\begin{tabular}{cccccccc}
\hline Ranking & TOPSIS & $\begin{array}{c}\text { Horas em } \\
\text { treinamento } \\
\text { EAD }\end{array}$ & $\begin{array}{c}\text { Horas em } \\
\text { treinamento } \\
\text { presencial }\end{array}$ & Ranking & TOPSIS & $\begin{array}{c}\text { Horas em } \\
\text { treinamento } \\
\text { EAD }\end{array}$ & $\begin{array}{c}\text { Horas em } \\
\text { treinamento } \\
\text { presencial }\end{array}$ \\
\hline $\mathbf{1}^{\mathbf{0}}$ & 0,91 & 141 & 1468 & $\mathbf{2 5 5}^{\mathbf{0}}$ & 0,28 & 47 & 988 \\
$\mathbf{2}^{\mathbf{o}}$ & 0,90 & 85 & 1198 & $\mathbf{2 5 6}^{\mathbf{0}}$ & 0,28 & 120 & 2008 \\
$\mathbf{3}^{\mathbf{0}}$ & 0,89 & 146 & 1777 & $\mathbf{2 5 7}^{\mathbf{0}}$ & 0,26 & 39 & 2247 \\
$\mathbf{4}^{\mathbf{0}}$ & 0,88 & 110 & 1663 & $\mathbf{2 5 8}^{\mathbf{o}}$ & 0,26 & 56 & 1639 \\
$\mathbf{5}^{\mathbf{0}}$ & 0,87 & 160 & 2409 & $\mathbf{2 5 9}^{\mathbf{0}}$ & 0,26 & 50 & 2293 \\
$\mathbf{6}^{\mathbf{0}}$ & 0,87 & 109 & 1701 & $\mathbf{2 6 0}^{\mathbf{0}}$ & 0,22 & 287 & 2703 \\
$\mathbf{7}^{\mathbf{0}}$ & 0,86 & 97 & 2450 & $\mathbf{2 6 1}^{\mathbf{0}}$ & 0,22 & 121 & 2169 \\
$\mathbf{8}^{\mathbf{0}}$ & 0,85 & 104 & 1691 & $\mathbf{2 6 2}^{\mathbf{0}}$ & 0,19 & 94 & 2350 \\
$\mathbf{9}^{\mathbf{0}}$ & 0,81 & 80 & 2169 & $\mathbf{2 6 3}^{\mathbf{0}}$ & 0,17 & 38 & 2453 \\
$\mathbf{1 0}^{\mathbf{0}}$ & 0,81 & 64 & 1655 & $\mathbf{2 6 4}^{\mathbf{0}}$ & 0,15 & 73 & 2807 \\
\hline
\end{tabular}

Fonte: Elaborado pelos autores.

Nota-se, a partir da Tabela 1, que a carga horária de treinamentos na modalidade presencial é muito superior a modalidade EAD em praticamente todos os casos, independente da posição no ranking TOPSIS desenvolvido. Esse dado reforça o fato de que, apesar de ter-se realizado mais de 1 milhão de horas em cursos à distância, o histórico recente da implementação dessa modalidade ainda apresenta baixo índice de procura pelas equipes em comparação as modalidades presenciais. As estatísticas descritivas mostradas na Tabela 2 permitem ainda observar que o volume mínimo de treinamento presencial é superior ao máximo em horas EAD. 
Tabela 2 - Estatísticas Descritivas - Gestores Pessoa Jurídica

\begin{tabular}{ccccc}
\hline Variável & Mínimo & Máximo & Média & Desvio Padrão \\
\hline Topsis & 0 & 1 &, 55 &, 139 \\
ID & 489 & 1312 & 905,83 & 121,838 \\
Sexo & 0 & 1 &, 57 &, 496 \\
Idade & 26 & 65 & 42,75 & 9,147 \\
TempoEmpr & 5 & 43 & 16,50 & 10,853 \\
HorasEAD & 11 & 337 & 107,16 & 57,833 \\
HorasPresen & 508 & 4638 & 1940,14 & 570,616 \\
\hline
\end{tabular}

Fonte: Dados da pesquisa

Na Tabela 3 são apresentados os coeficientes da relação entre o ranking TOPSIS e as demais variáveis de pesquisa. Analisando-se a regressão, verifica-se inicialmente que o ranking TOPSIS gerado na pesquisa está alinhado com o índice de desempenho $(0,912 ; \mathrm{p}<0,01)$ divulgado pela empresa, apesar dos itens e o método de cálculo não serem os mesmos.

Tabela 3: Regressão linear para os dados dos gestores pessoa jurídica

\begin{tabular}{|c|c|c|c|}
\hline $\begin{array}{c}\text { Variável } \\
\text { Dependente: Topsis }\end{array}$ & $\begin{array}{c}\text { Coef. } \\
\text { Padronizado }\end{array}$ & Teste $t$ & Sig. \\
\hline Constante & & $-33,038$ &, $000 * *$ \\
\hline ID & ,912 & 110,731 &, $000 * *$ \\
\hline Sexo &,- 017 & $-2,040$ &, $041 *$ \\
\hline Idade &, 112 & 7,536 &, $000 * *$ \\
\hline TempoEmpr &,- 127 & $-8,488$ &, $000 * *$ \\
\hline HorasEAD &, 022 & 2,539 &, $011 *$ \\
\hline HorasPresen &,- 024 & $-2,991$ &, $003 *$ \\
\hline Controle Fixo & & Sim & \\
\hline $\mathrm{R}^{2}$ & & 0,832 & \\
\hline $\mathrm{R}^{2}$ ajustado & & 0,828 & \\
\hline ANOVA & & $0,000 *$ & \\
\hline Durbin-Watson & & 1,739 & \\
\hline $\mathrm{N}^{\circ}$ Observações & & 264 & \\
\hline
\end{tabular}

A relação da idade com o ranking TOPSIS mostrou-se positiva e significativa $(0,112$; $\mathrm{p}<0,01$ ), isto contraria as expectativas anteriores. Por sua vez, o tempo de empresa confirmou os indícios levantados na análise da correlação, pois apresenta coeficiente negativo e significativo $(-0,127 ; \mathrm{p}<0,01)$, apesar desse último ter sido consideravelmente inferior ao da correlação da associação direta entre as variáveis $(-0,404 ; \mathrm{p}<0,01)$.

Conforme previsto pela literatura anterior, a maior carga horária em treinamentos à distância (EAD) apresenta impacto positivo e significativo $(0,022 ; p<0,01)$ no ranking TOPSIS desenvolvido para os gestores de pessoa jurídica. Esse resultado mostra uma tendência de mudança para a inovação e sustentabilidade organizacional nos processos da instituição financeira estudada, considerando-se que estudos anteriores como o de Pires e Marcondes (2004) evidenciavam um cenário de desinteresse pela gestão do conhecimento, baixo nível de inovação e aprendizagem organizacional e manipulação de dados de clientes. Diante disso, confirma-se a hipótese proposta $(\mathrm{H} 2)$ de que os treinamentos EAD ampliam o desempenho dos gestores.

Por outro lado, a regressão apresentou resultados negativos e significativos para o treinamento presencial $(-0,024 ; \mathrm{p}<0,01)$ em relação ao ranking TOPSIS de desempenho. Isto contraria os achados na literatura (FREIRE et al., 2017; MENEZES et al., 2018) e rejeita a hipótese $\mathrm{H}_{1}$ desse estudo. Segundo entrevista realizada com três desses gestores, algumas explicações podem estar subjacentes a tais resultados, tais como o fato de que o profissional 
para receber os treinamentos presenciais precisa se ausentar de suas atividades para deslocarse até o centro de treinamentos da instituição, ou que os cursos não possuem a mesma objetividade que os treinamentos e o material digital disponibilizado nos treinamentos EAD. Outro motivo, segundo eles, é que o material do treinamento EAD fica disponível no dia a dia de trabalho, enquanto o treinamento presencial é apenas teórico e quando retornam para a prática nem sempre recordam de todas as informações recebidas.

Por fim, argumentaram que o fluxo de atendimentos e demanda dos clientes apresenta sazonalidade ao longo do mês, é intenso no período do dia $1^{\circ}$ ao dia 10 e baixo nos demais, logo, treinamentos EAD podem ser feitos em períodos à escolha do gestor enquanto os presenciais são determinados pela empresa.

$\mathrm{Na}$ sequência analisa-se os mesmos procedimentos metodológicos, contudo, foram adotados para a amostra dos gerentes pessoa física e os resultados são apresentados na próxima seção.

\subsection{Gerentes Pessoa Física $(\mathrm{N}=823)$}

Seguindo-se a mesma linha de raciocínio adotada para apresentar os dados da seção 4.1, os dados indicados na Tabela 4 mostram os 10 melhores (mais próximos de 1) e os 10 piores (mais distantes de 1) entre os 823 gestores pessoa física analisados, assim como o total de horas dedicadas a treinamentos na modalidade EAD e na modalidade presencial.

Tabela 4 - Ranking dos gestores pessoa física baseado na técnica TOPSIS

\begin{tabular}{cccccccc}
\hline Ranking & TOPSIS & $\begin{array}{c}\text { Horas em } \\
\text { treinamento } \\
\text { EAD }\end{array}$ & $\begin{array}{c}\text { Horas em } \\
\text { treinamento } \\
\text { presencial }\end{array}$ & Ranking & TOPSIS & $\begin{array}{c}\text { Horas em } \\
\text { treinamento } \\
\text { EAD }\end{array}$ & $\begin{array}{c}\text { Horas em } \\
\text { treinamento } \\
\text { presencial }\end{array}$ \\
\hline $\mathbf{1}^{\mathbf{0}}$ & 0,96 & 63 & 493 & $\mathbf{8 1 4}^{\mathbf{0}}$ & 0,31 & 52 & 374 \\
$\mathbf{2}^{\mathbf{o}}$ & 0,95 & 77 & 1118 & $\mathbf{8 1 5}^{\mathbf{0}}$ & 0,30 & 169 & 948 \\
$\mathbf{3}^{\mathbf{o}}$ & 0,95 & 111 & 633 & $\mathbf{8 1 6}^{\mathbf{0}}$ & 0,30 & 123 & 548 \\
$\mathbf{4}^{\mathbf{o}}$ & 0,95 & 89 & 550 & $\mathbf{8 1 7}^{\mathbf{0}}$ & 0,29 & 75 & 297 \\
$\mathbf{5}^{\mathbf{0}}$ & 0,94 & 72 & 923 & $\mathbf{8 1 8}^{\mathbf{o}}$ & 0,28 & 178 & 595 \\
$\mathbf{6}^{\mathbf{0}}$ & 0,94 & 244 & 1058 & $\mathbf{8 1 9}^{\mathbf{o}}$ & 0,28 & 95 & 266 \\
$\mathbf{7}^{\mathbf{0}}$ & 0,94 & 126 & 1250 & $\mathbf{8 2 0}^{\mathbf{0}}$ & 0,28 & 73 & 309 \\
$\mathbf{8}^{\mathbf{o}}$ & 0,94 & 90 & 504 & $\mathbf{8 2 1}^{\mathbf{0}}$ & 0,26 & 90 & 375 \\
$\mathbf{9}^{\mathbf{o}}$ & 0,94 & 103 & 1828 & $\mathbf{8 2 2}^{\mathbf{o}}$ & 0,25 & 86 & 544 \\
$\mathbf{1 0}^{\mathbf{o}}$ & 0,93 & 73 & 191 & $\mathbf{8 2 3}^{\mathbf{0}}$ & 0,23 & 139 & 238 \\
\hline
\end{tabular}

Fonte: Elaborado pelos autores.

Novamente é possível observar a informação mostrada por meio da Tabela 4, de que o número de horas/treinamentos na modalidade presencial de gestores pessoa física é superior a modalidade EAD em praticamente todos os casos, independente da posição no ranking desenvolvido. Tal informação corrobora e confirma a situação de prevalência da modalidade de ensino presencial para treinamento de equipes. As estatísticas descritivas da Tabela 5 permitem também observar que o volume mínimo de treinamento presencial é superior ao máximo em horas EAD.

Além disso, a estatística descritiva mostra que, para os gestores pessoa física, a Instituição apresenta o treinamento presencial como carro chefe, pois o desvio padrão desta variável é o maior entre todas as demais, o que indica heterogeneidade nos treinamentos oferecidos de acordo com a necessidade dos colaboradores, até que se atinjam os objetivos dos treinamentos.

Outra importante verificação é que a idade dos gestores pessoa física está entre 26 e 67 anos, o que denota as características de políticas de treinamento visando a manutenção da 
qualidade e atualização dos serviços prestados, independente da idade e do tempo de empresa dos colaboradores.

Tabela 5 - Estatísticas Descritivas - Gestores Pessoa Física

\begin{tabular}{ccccc}
\hline Variável & Mínimo & Máximo & Média & Desvio Padrão \\
\hline Topsis &, 23 &, 96 &, 6246 &, 12776 \\
ID & 716,28 & 1250,50 & 996,0632 & 90,41651 \\
Sexo & 0 & 1,00 &, 6719 &, 46980 \\
Idade & 26 & 67,00 & 44,0073 & 9,63229 \\
TempoEmpr & 5 & 46,00 & 17,1519 & 10,89041 \\
HorasEAD & 6 & 370,00 & 109,7193 & 59,36661 \\
HorasPresen & 70 & 3277,00 & 468,1665 & 360,57546 \\
\hline
\end{tabular}

Fonte: Dados da pesquisa

Os resultados da regressão apresentados na Tabela 6 indicam que o ranking TOPSIS gerado na pesquisa está alinhado com o índice de desempenho $(0,979 ; \mathrm{p}<0,01)$ divulgado pela empresa, apesar dos itens e método de cálculo não serem os mesmos. Tal resultado é semelhante ao obtido para os gestores pessoa jurídica.

Tabela 6: Regressão linear para os dados dos gestores pessoa física

\begin{tabular}{|c|c|c|c|}
\hline $\begin{array}{c}\text { Variável } \\
\text { Dependente: Topsis }\end{array}$ & $\begin{array}{c}\text { Coef. } \\
\text { Padronizado }\end{array}$ & Teste $\mathbf{t}$ & Sig. \\
\hline Constante & & $-195,717$ &, $000 * *$ \\
\hline ID & ,979 & 439,278 &, $000 * *$ \\
\hline Sexo & ,008 & 3,443 &, $001 * *$ \\
\hline Idade &, 002 & 0,602 &, 547 \\
\hline TempoEmpr &,- 010 & $-2,534$ &, $011 * *$ \\
\hline HorasEAD &,- 009 & $-3,730$ &, $000 *$ \\
\hline HorasPresen &,- 011 & $-5,018$ &, $000 *$ \\
\hline Controle Fixo & & Sim & \\
\hline $\mathrm{R}^{2}$ & & 0,0960 & \\
\hline $\mathrm{R}^{2}$ ajustado & & 0,960 & \\
\hline ANOVA & & $0,000^{*}$ & \\
\hline Durbin-Watson & & 2,078 & \\
\hline $\mathrm{N}^{\circ}$ Observações & & 823 & \\
\hline
\end{tabular}

Por outro lado, para esse grupo de gestores, ambas às modalidades de treinamento apresentaram resultados negativos sobre o desempenho individual (HorasEAD: -0,009; $p<0,01$ e HorasPresen: -0,011; $\mathrm{p}<0,01)$. Esses achados contrariam as evidências da literatura (Freire et al., 2017; Menezes et al., 2018) e rejeitam ambas as hipóteses H1 e H2 desse estudo.

Cinco gestores pessoa física foram questionados em relação a esse resultado e informaram que, a carga horária dos gestores pessoa jurídica é de oito horas diárias, com intervalo de 1 hora, por sua vez, a carga horária dos gestores pessoa física é de seis horas com intervalo de 30 minutos. Como a empresa fica aberta para atendimento ao público durante cinco horas por dia, eles acreditam que os gestores pessoa jurídica possuam mais tempo para se dedicarem aos treinamentos durante a jornada de trabalho, sem interrupções pela chegada de clientes e com maior concentração, em consequência, possibilita maior aprendizado e melhor desempenho.

Outros fatores que podem explicar a causa do efeito negativo do EAD sobre o desempenho dos gestores pessoa física divergente daqueles da pessoa jurídica, são descritos no estudo de Brauer e Albertin (2010) e estão relacionados com as resistências à essa modalidade. Esses autores, com base na literatura anterior, elencaram a indisciplina e mal gerenciamento do tempo, a procrastinação, o pouco conhecimento e habilidade em TI e influência social, entre 
outros, como fatores de resistência ao uso de EAD em treinamentos corporativos e, por consequência, como justificativa para seu baixo efeito sobre o desempenho.

Galusha (1997) afirma que no ambiente organizacional, um fator predominante para o sucesso dos treinamentos é a motivação do empregado, em especial em cursos EAD, por isso conhecer as características do perfil individual de cada grupo de alunos é essencial para o alcançar os objetivos.

Nesse sentido, Carvalho e Abbad (2006) apresentam um modelo para avaliação de desempenho em amplitude e profundidade no qual, além de analisar dados do público-alvo, neste caso gestores pessoa física e jurídica, também avaliam os objetivos do treinamento, pois somente é possível afirmar que o treinamento não obteve êxito, comparando-se resultados obtidos com os objetivos previstos inicialmente.

Portanto, apesar de avançar na literatura por analisar os efeitos de programas e seu resultado efetivo sobre o desempenho de gestores, esse estudo apresenta algumas limitações que serão apresentadas a seguir.

\section{CONSIDERAÇÕES FINAIS}

Este trabalho teve como objetivo investigar a relação entre os treinamentos presenciais e à distância oferecidos por uma universidade corporativa e o desempenho individual dos gestores da organização. Os principais resultados permitem constatar a importância da Universidade Corporativa na estratégia de negócios da organização pesquisada, destacar a priorização inicial do ensino presencial, a recente inserção da modalidade à distância e seu efeito positivo para um dos grupos da amostra e negativo para o outro.

Pode-se inferir também que, apesar de inúmeras vantagens existentes na adoção de cursos e treinamentos na modalidade a distância pelas universidades corporativas, principalmente financeiras e espaço-temporais, nem sempre essa modalidade apresenta resultados positivos sobre o desempenho individual de gestores.

Mesmo apresentando avanços em relação à estudos anteriores, essa pesquisa apresenta limitações. Primeiramente com relação aos dados coletados, pois a carga horária de treinamento considerada para cada funcionário é a total, desde que o mesmo ingressou na empresa. Logo, como a modalidade EAD é recente (cinco anos), a carga horária presencial tende a ser superior.

Outro fator limitador foi observado por Carvalho e Abbad (2006) quanto à avaliação dos treinamentos em relação aos seus objetivos. Os treinamentos foram analisados apenas a partir de sua carga horária, sem distinção de objetivos, logo, é provável que nem todos guardem relação com os objetivos comerciais e, portanto, impactem diretamente no índice de desempenho medido pela empresa. Nesse sentido, recomenda-se que pesquisas futuras analisem o impacto de cada curso nos resultados relacionados diretamente com seus objetivos.

É importante destacar também que esse estudo analisou dados de uma única Universidade Corporativa, empresa e segmento. Desse modo, sugere-se estudos multicascos em que se possa comparar os resultados dos treinamentos presenciais e a distância em empresas de diversos tamanhos, mercados, culturas e níveis de ciclo de vida organizacional.

Ademais, sugere-se a realização de pesquisas empíricas que situem as universidades corporativas em uma abordagem de instituições, de forma a observar características macros que impulsionam a criação e o desenvolvimento das mesmas, como por exemplo, pesquisar os fatores ligados às forças coercitivas, normativas e miméticas estudadas por Dimaggio e Powell (1983) e a forma como influenciam a dinâmica destas instituições.

Pode-se também partir da investigação do papel comportamental das universidades corporativas, como o impacto na satisfação e motivação dos gestores, a influência no desempenho de seus papeis, os estímulos sociais percebidos, dentre outros fatores. Em uma perspectiva da eficácia, pode-se analisar a capacidade substitutiva, complementar ou suplementar dos conteúdos estudados nas UCs e compará-los às matrizes curriculares dos 
cursos regulares das instituições de ensino públicas ou privadas, e desta forma, compreender seu papel e aderência aos aspectos didáticos que se espera de um modelo de ensino.

$\mathrm{Na}$ direção da pesquisa de Kraemer (2018), sugere-se estudos sob o viés econômico, e neste contexto, analisar a estrutura operacional e administrativa das universidades corporativas e compará-las ao ciclo de vida das empresas, de forma a evidenciar o efeito dos fatores econômicas na composição e manutenção das UCs.

Por fim, decorrente do evento da pandemia de coronavírus, torna-se relevante realizar estudos dessa natureza nesse período, pois os treinamentos à distância e o trabalho remoto aumentaram de forma significante e podem ter influenciado as relações sociais.

\section{REFERÊNCIAS}

ABEL, A., LI, J. Exploring the corporate university phenomenon: development and implementation of a comprehensive survey. Human Resource Development Quarterly, v. 23, n. 1, p. 103-128, 2012. doi: https://doi.org/10.1002/hrdq.21122

ALAGARAJA, M.; LI, J. Utilizing institucional perspectives to investigate the emergence, rise and (relative) decline of corporative universities. Human Resource Development International, v. 18, n. 1, p. 4-23, 2015. doi: https://doi.org/10.1080/13678868.2014.979003

ALLEN, M. What is a corporate university, and why should an organization have one? in: ALLEN, M. The corporate university handbook: designing, managing and growing a successful program. Nova York: Amacom, p. 1-12, 2002.

ALAGARAJA, M.; LI, J. Utilizing institutional perspectives to investigate the emergence, rise, and (relative) decline of corporate universities. Human Resource Development International, v. 18, n. 1, p. 4-23, 2015. doi: https://doi.org/10.1080/13678868.2014.979003

ANTONELLO, C. S.; GODOY, A. S. A encruzilhada da aprendizagem organizacional: uma visão multiparadigmática. RAC, v. 14, n. 2, p. 310-332, 2010. doi: https://doi.org/10.1590/S1415-65552010000200008

ARAUJO, L.; EASTERBY-SMITH, M. Aprendizagem organizacional: oportunidades e debates atuais. In: Easterby-Smith, M.; Burgoyne, J.; Araújo, L. (Coord.). Aprendizagem organizacional e organização de aprendizagem: desenvolvimento na teoria e na prática. São Paulo: Atlas, 2001.

ARGOTE, L.; MIRON-SPEKTOR, E. Organizational learning: from experience to knowledge. Organization Science, v. 22, n. 5, p. 1123-1137, 2011. doi: https://doi.org/10.1287/orsc.1100.0621

AVELINO, G. I. B. Relação entre competências gerenciais e desempenho organizacional: um estudo de caso em instituição financeira. 2016. Dissertação (Mestrado em Administração) - Programa de Pós-Graduação em Administração da Pontifícia Universidade Católica de Minas Gerais - PUC-MG, 2016.

BEUREN, I. M.; ROTH, T. C.; ANZILAGO, M. Efeitos da aplicação do princípio da controlabilidade no desempenho gerencial mediada pelo conflito e ambiguidade de papeis. Revista Universo Contábil, v. 13, n 3, p. 06-28, 2017. doi: 10.4270/ruc.2017316 
BRAUER, M.; ALBERTIN, L. A. Educação corporativa a distância: por que tanta resistência? Redige, v. 1, n. 1, p. 141-157, 2010. Recuperado de https://www.researchgate.net/profile/Alberto_Albertin/publication/280839302_Educacao_cor porativa_a_distancia_por_que_tanta_resistencia/links/55c8d3f508aebc967df90492.pdf

BOERNER, C.; MACHER, J.; TEECE, D. A review and assessment of organizational learning in economic theories. In: M. Dierkes, A. Berthoin Antal, J. Child, \& I. Nonaka (Orgs.), The handbook of organizational learning and knowledge, p. 89-117. Oxford: Oxford University Press, 2001.

BULGURCU, B. K. Application of TOPSIS technique for financial performance evaluation of technology firms in Istanbul stock exchange market. Procedia-Social and Behavioral Sciences, v. 62, p. 1033-1040, 2012. doi: 10.1016/j.sbspro.2012.09.176

CAPPIELLO, G.; PEDRINI, G. The performance evaluation of corporate universities. Tertiary Education and Management, v. 23, n. 3, p. 304-317, 2017. doi: https://doi.org/10.1080/13583883.2017.1329452.

CARVALHO, r. s.; ABBAD, G. Avaliaçăo de Treinamento a Distância: Reação, Suporte à Transferência e Impactos no Trabalho. RAC, v. 10, n. 1, p. 95-116, 2006. doi: http://dx.doi.org/10.1590/S1415-65552006000100006

CASTRO, C. M.; EBOLI, M. Universidade corporativa: gênese e questões críticas rumo à maturidade. RAE, v. 53, n. 4, p. 408-414, 2013. doi: http://dx.doi.org/10.1590/S003475902013000400008

COSTA, F. R.; PELISSARI, A. S. Imagem corporativa: fatores influenciadores sob a ótica dos discentes da educação à distância. Brazilian Business Review, v. 14, n.1, p. 110-132, 2017. doi: http://dx.doi.org/10.15728/bbr.2017.14.1.6

DAVEL, E. P. B.; OLIVEIRA, C. A. A reflexividade intensiva na aprendizagem organizacional: uma autoetnografia de práticas em uma organização educacional. Organizações \& Sociedade - $\mathbf{O \& S}$, v. 25, n. 85, p. 211-228, 2018. Recuperado de https://rigs.ufba.br/index.php/revistaoes/article/view/15877

DAVENPORT, T. H.; PRUSAK, L. Conhecimento empresarial: como as organizações gerenciam o seu capital intelectual. Rio de Janeiro: Campus, 1998.

DIAKOULAKI, D.; MAVROTAS, G.; PAPAYANNAKIS, L. Determining objective weights in multiple criteria problems: the critic method. Computers \& Operations Research, v. 22, $n$. 7, p. 763-770, 1995. doi: https://doi.org/10.1016/0305-0548(94)00059-H

DiMAGGIO, P.; POWELL, W. The iron cage revisited: collective rationality and institutional isomorphism in organizational fields. American Sociological Review, v. 48, n. 2, p. 147-160, 1983. doi: https://doi.org/10.2307/2095101

DURAN, D. A educação a distância no processo de formação continuada da administração pública: as contribuições da revista do serviço público. Revista do Serviço Público, v. 68, n. 3, p. 705-736, 2017. doi: https://doi.org/10.21874/rsp.v6 
EASTERBY, S. M.; PRIETO, I. M. Dynamic Capabilities and Knowledge Management: an Integrative Role for Learning? British Journal of Management, v. 19, n. 3, p. 235-249, 2008. doi: https://doi.org/10.1111/j.1467-8551.2007.00543.x

EBOLI, M. (2011). Sistema de educação corporativa e a EAD. In F. M. Litto \& M. Formiga (Orgs.), Educação a distância: o estado da arte (p. 189-197). São Paulo: Pearson, 2011.

EBOLI, M. Educação corporativa nos novos cenários empresariais. GV-executivo, v. 15, n. 2, p. 20-24, 2016.

ESTEVES, L.P.; MEIRIÑO, M.J. A educação corporativa e a gestão do conhecimento. In: CONGRESSO NACIONAL DE EXCELÊNCIA EM GESTÃO, 11., 2015. Rio de Janeiro. Anais [...]. Rio de Janeiro: FIRJAN, 2015. p. 1-12.

FÁVERO, L. P.; FÁVERO, P. Análise de dados: modelos de regressão com Excel®, Stata ${ }^{\circledR}$ e SPSS $\AA^{2}, v .1$. Campus: Elsevier Brasil, 2016.

FLEURY, M. T. L.; RUAS, R. Competências: conceitos, métodos e experiências. São Paulo: Atlas, 2008.

FREIRE, T. E.; SERACHINI, M. R.; BAZILIO, V.; FREITAS, E. Gestão de pessoas: uso da educação a distância como ferramenta nos treinamentos e desenvolvimentos corporativos. Revista de Ciências Gerenciais, v. 21, n. 34, p. 114-120, 2017. doi: http://dx.doi.org/10.17921/1415-6571.2017v21n34p114-120

FREITAS, P. F. P.; ODELIUS, C. C. Competências gerenciais: uma análise de classificações em estudos empíricos. Cad. EBAPE. BR, v. 16, n. 1, p. 35-49, 2018. Recuperado de http://bibliotecadigital.fgv.br/ojs/index.php/cadernosebape/article/view/59497/71091

Galusha, J. M. Barriers to learning in distance education. Interpersonal Computing and Technology, v. 5, n. 3, p. 6-14, 1997. Retrieved from https://www.learntechlib.org/p/85240/

GONÇALVES, W.A.; ANDRADE, W. M.; CORRÊA, D. A.; RIBEIRO, G. G. Confrontando o conceito de competências pela sua diversidade e aplicação: um olhar entre a teoria e a prática. Pretexto, v. 18, n. 4, p. 114-128, 2017. doi: http://dx.doi.org/10.21714/pretexto.v18i4.2722

HALL M. (2008). The effect of comprehensive performance measurement systems on role clarity, psychological empowerment and managerial performance. Accounting, Organizations and Society, v. 33, n. 2-3, p. 141-163, 2008. doi: https://doi.org/10.1016/j.aos.2007.02.004

HELFAT, C.; PETERAF, M. (2009). Understanding dynamic capabilities: progress along a developmental path. Strategic Organization, v. 7, n. 1, p. 91-102, 2009. doi: $10.1177 / 1476127008100133$

IMAMOGLU, S.Z.; INCE, H.; KESKIN, H.; KARAKOSE, M.A.; GOZUKARA, E. The role of leadership styles and organizational learning capability on firm performance, Journal of Global Strategic Management, v. 9, n. 1, p. 113-124, 2015. doi: 10.20460/JGSM.2015915633 
JACOMOSSI, R. R.; DEMAJOROVIC, J. Fatores determinantes da aprendizagem organizacional para a inovação ambiental: um estudo multicaso. RAC, v. 21, n. 5, p. 685-709, 2017. doi: http://dx.doi.org/10.1590/1982-7849rac2017160281

JOIA, L. A.; Da Costa, M. D. F. C. Fatores-chave de sucesso no treinamento corporativo a distância via web. Revista de Administração Pública, v. 41, n. 4, p. 607-638, 2007. doi: https://doi.org/10.1590/S0034-76122007000400002.

KRAEMER, R. Modelo de maturidade do sistema de educação corporativa. Dissertação. (Mestrado em Engenharia e Gestão do Conhecimento). Programa de Pós-Graduação em Engenharia e Gestão do Conhecimento. Universidade Federal de Santa Catarina. FlorianópolisSC, 2018.

LEAL, A. P. Treinamento, desenvolvimento e educação corporativa: a qualificação como instrumento de subordinação do trabalho ao capital. In Soboll, L. A., Ferraz, D. L. S. Gestão de pessoas-Armadilhas da organização do trabalho. São Paulo: Atlas, 2014.

LEE, S.; RITTINER, F.; SZULANSKI, G. The past, present, and future of organizational learning research: a conversation with professor Linda Argote. Journal of Management Inquiry, v. 25, n. 1, p. 85-92, 2015. doi: https://doi.org/10.1177/1056492615576974

LYTOVCHENKO, I. Corporate university as a form of employee training and development in american companies. Advanced Education, v. 5, p. 35-41, 2016. doi: 10.20535/24108286.62280 .

MACHADO, D. P.; MORAES, M. G. S. Educação à distância: fundamentos, tecnologias, estrutura e processos de ensino e aprendizagem. São Paulo: Érica, 2015.

MAGLIONE, R.; PASSIANTE, G. The stakeholder university as dynamic learning network: The Finmeccanica case. In A. Romano \& G. Secundo (Eds.), Dynamic learning networks: Model and cases in action (pp. 95-120). New York, NY: Springer, 2009.

MALVEZZI, S. Do taylorismo ao comportamentalismo: 90 anos de desenvolvimento de recursos humanos. BOOG, GG Manual de Treinamento e Desenvolvimento ABTD. $2^{\mathrm{a}}$ ed. São Paulo: Mackron Books, 1994.

MEISTER, J. Educação corporativa: a gestão do capital intelectual através das universidades corporativas. São Paulo: Makron Books, 1999.

MENEZES, R. F.; PASSOS, J. C.; OLIVEIRA, M. F.; BARBOSA, I.; RODRIGUES, H. G. Análise do processo de treinamento de uma universidade corporativa pela perspectiva da abordagem sistêmica de treinamento. Universitas: Gestão e TI, v. 7, n. 1-2, p. 31-39, 2018. doi: http://dx.doi.org/10.5102/un.gti.v7i1.3590

MERCHANT, K. A.; VAN DER STEDE, W. A. Management control systems: performance measurement, evaluation and incentives. New York: Pearson, 2007.

MOSCARDINI, T. N.; Klein, A. Educação Corporativa e Desenvolvimento de Lideranças em Empresas Multisite. Revista de Administração Contemporânea, v. 19, n. 1, p. 84-106, 2015. doi: http://dx.doi.org/10.1590/1982-7849rac20151879 
MUNGANIA, P. The seven e-learning barriers facing employees. The Masie Centre, 2003.

NASCIMENTO, R. J. C. Processo de aprendizagem organizacional no desenvolvimento de competências organizacionais. Dissertação (Mestrado em Administração). Programa de PósGraduação em Administração - PPGA, Universidade de Caxias do Sul - Caxias do Sul-RS, 2017.

PAVITT, K. The objectives of technology policy. Science and Public Policy, v. 14, n. 4, p. 182-188, 1987. doi: https://doi.org/10.1093/spp/14.4.182

RUGGIERO, S. Educação Corporativa: a medida da adequação das universidades corporativas. Revista de Ciências Gerenciais, v. 10, n. 2, p. $51.57,2006$. doi: http://dx.doi.org/10.17921/1415-6571.2006v10n12p51-57

RYAN, L.; PRINCE, C.; TURNER, P. The changing and developing role of the corporate university post-millennium. Industry \& Higher Education, v. 20, n. 3, p. 167-174, 2015. doi: https://doi.org/10.5367/ihe.2015.0256

SCHARDOSIM, P. R.; GOMES Jr. W. V.; TORQUATO, M.; LAPOLLI, E. F. Aprendizagem organizacional: estudo de caso no setor de geoprocessamento em uma organização intensiva em conhecimento. Espacios, v. 38, n. 1, p. 9, 2017. Recuperado de http://www.revistaespacios.com/a17v38n01/a17v38n01p09.pdf

SILVA, T. C.; FREIRE, P. S. Universidade corporativa em rede: diretrizes que devem ser gerenciadas para a implantação do modelo. Espacios, v. 38, n. 10, p. 13, 2017. Recuperado de http://www.revistaespacios.com/a17v38n10/a17v38n10p13.pdf

SIMONS, R. Levers of Organization Design: how managers use accountability systems for greater performance and commitment. Cambridge, MA: Harvard Business School Press, 2005 .

TZENG, G. H.; HUANG, J. J. Multiple attribute decision making: methods and applications. Chapman and Hall/CRC, 2011.

VIDAL, F. R. P. Concepções de educação corporativa: um estudo da produção discente da pós-graduação em duas áreas do conhecimento: educação e administração (1990-2014). Dissertação (Mestrado em Educação). Programa de Pós-Graduação Stricto Sensu Universidade Católica de Santos. Santos, Brasil, 2017.

WANG, G.; LI, J.; X. Qiao; SUN, J. Understanding the corporate university phenomenon: A human capital theory perspective. International Journal of Human Resources Development and Management, v. $10, \quad$ n. $2, \quad$ p. 182-204, 2010. doi: https://doi.org/10.1504/IJHRDM.2010.031443

YIN, R. K. Estudo de caso: planejamento e métodos. 5.ed. Bookman: 2015.

ZERBINI, T.; NASCIMENTO, S. B.; MENESES, P. P. M.; ABBAD, G. Percepções sobre educação a distância: limitações e restrições à implantação da Universidade Corporativa do Banco Central do Brasil. Anais[...]. ENCONTRO DA ANPAD, ENANPAD, 30, Salvador-BA Brasil, 2006. 